\title{
Correspondence re "Evidence-based scientific exercise guidelines for adults with spinal cord injury: an update and new guideline"
}

\author{
Sean M. Tweedy ${ }^{1} \cdot$ Emma M. Beckman ${ }^{1} \cdot$ Mark J. Connick $^{1} \cdot$ Timothy J. Geraghty $^{2} \cdot$ Daniel Theisen $^{3} \cdot$ Claudio Perret $^{4}$. \\ Walter R. Thompson ${ }^{5} \cdot$ Yves C. Vanlandewijck ${ }^{6}$
}

Received: 24 November 2017 / Accepted: 27 November 2017 / Published online: 18 January 2018

(c) International Spinal Cord Society 2018

New exercise guidelines for adults with spinal cord injury (SCI) have been published [1]. There are important differences between them and the recently published Exercise and Sports Science Australia (ESSA) Position Statement on exercise and SCI authored by ST, EB, TG, DT, CP and YV [2] including:

ESSA recommends a single exercise prescription that will confer good health, while the New Guidelines (NG) recommend two different prescriptions: one to achieve fitness benefits and a separate one to achieve cardiometabolic health benefits;

the volume of aerobic exercise recommended by ESSA is $150 \mathrm{~min}$ of moderate-intensity exercise (MIE)/wk, considerably larger than the NG, which recommend at least $40 \mathrm{~min}$ of MIE/wk (fitness benefits) or at least $90 \mathrm{~min}$ of MIE/wk (cardiometabolic health benefit);

the NG recommendations are presented as "minimum thresholds", implying that the sub-threshold volumes will not confer fitness/health benefits. In contrast, ESSA recognises that many "Beginning Clients" will initially be

Sean M. Tweedy

s.tweedy@uq.edu.au

1 School of Human Movement and Nutrition Sciences, The University of Queensland, St Lucia, Australia

2 Queensland Spinal Cord Injuries Service, Princess Alexandra Hospital, Metro South Health, Brisbane, Australia

3 Sports Medicine Research Laboratory, Luxembourg Institute of Health, Grand-Duchy of Luxembourg, Luxembourg

4 Institute of Sports Medicine, Swiss Paraplegic Centre Nottwil, Nottwil, Switzerland

5 College of Education and Human Development, Georgia State University, Atlanta, USA

6 Faculty of Kinesiology and Rehabilitation Sciences, Katholieke Universiteit Leuven, Leuven, Belgium unable to safely meet the recommended volume and these clients will derive benefit from very small, progressive increases in exercise volume (e.g. $5 \mathrm{~min} /$ day).

The discrepancies prompted us to evaluate the primary evidence supporting the NG. Unfortunately, this task was made difficult, because the primary sources are not included in the reference list of the NG or the systematic review (SR) on which the NG are based [3]. Instead, they are in an electronic supplement on the website of the Journal Neurology (http://www.neurology.org/content/89/7/736/suppl/ DC1, retrieved on 3rd Nov 2017). Specifically, Table 2 in the NG directs the reader to Tables 3 and 4 of the SR together with, respectively, Supplementary Tables e-10 and e-11. The Supplementary Tables must be cross-linked to Tables e-18 and e-19, where brief references are found that can be used to locate the full references associated with Table e-5. In the interests of transparency and permitting critique, we believe that the primary Level 1 and 2 sources underpinning the NG should be made more readily available, if not in the reference list, then at least in a supplement to the NG.

Based on our reading of the Supplementary Tables of the $\mathrm{SR}$, the guideline for the minimum volume of aerobic activity required to improve fitness, $-2 \times 20 \mathrm{~min} /$ week of moderate-intensity exercise-is based on the evidence from six exercise training studies [4-9]. Each of these studies had a control group and the remainder of the participants completed a specific exercise prescription, the aerobic training component of which is summarised as follows:

- * Study \#1 [5]: 9mo; 2x/week at 70\% $\mathrm{HR}_{\max }$ (moderate intensity); Initially $2 \times 5-10 \mathrm{~min} / \mathrm{session}$, progressing to $1 \times 15-30 \mathrm{~min} / \mathrm{session}$;

- "Study \#2 [4]: 16 weeks; 2x/week at RPE 3-6 (moderate to vigorous intensity); $1 \times 20 \mathrm{~min} / \mathrm{session}$;

- Study \#3 [6]: 24 weeks; 3x/week; Four groups completing different volumes, from $40 \mathrm{~min} / \mathrm{session} @$ 
$70 \% \mathrm{VO}_{2 \text { peak }}$ (highest volume) to $20 \mathrm{~min} / \mathrm{session} @ 50 \%$ $\mathrm{VO}_{2 \text { peak }}$ (lowest volume);

- Study \#4 [7]: 6 weeks; 3x/week; progressing from $70 \%$ $\mathrm{HR}_{\max }$ (moderate intensity) to $80 \% \mathrm{HR}_{\max }$ (vigorous intensity). Six intervals of $6 \mathrm{~min}$ exercise, with 1 min recovery (42 min total);

- Study \#5 [8]: 12 weeks; 3x/week at 50-65\% of HRR (moderate to vigorous intensity); progressing from 20 to $37.5 \mathrm{~min} / \mathrm{session}$;

- Study \#6 [9]: wheelchair basketballers training/playing $\sim 5 \mathrm{~h} /$ week completed an additional $5 \times 30 \mathrm{~min} /$ week for 8 weeks at $80 \% \mathrm{HR}_{\text {peak }}$ (vigorous)

*These studies included a resistance training component that is not reported here.

Each of these studies report positive cardiorespiratory changes in response to these various exercise prescriptions and provide sound evidence that upper body aerobic exercise - with or without resistance training - can improve cardiorespiratory fitness in adults with SCI. However, none of these studies were designed to identify a minimum threshold and consequently, none of them provides Level 1 or 2 evidence in support of the notion that individuals with an SCI must complete a minimum of 40 min per week of moderateintensity activity in order to improve cardiorespiratory fitness. Of the six studies, the one by Taylor and collegues [9] highlights the disconnect between the primary evidence and the NG [9]. In Taylor's study, participants already completing $5 \mathrm{~h}$ of wheelchair basketball per week increased their aerobic capacity by undertaking an additional $2.5 \mathrm{~h} /$ week of vigorous intensity exercise for 8 weeks. This study does not provide Level 1 or 2 evidence to support the aerobic recommendation for fitness benefit in the NG.

In fact, we posit that there is no primary Level 1 or Level 2 evidence to support any of the minimum exercise thresholds recommended in the NG for fitness or health. This is because all the level 1 and 2 studies cited in the SR are designed to evaluate the fitness and health responses of participants with SCI to a particular prescription; they are not designed to identify minimum thresholds for fitness and health benefit. Furthermore, since Item 12, Domain 3 of the AGREE reporting checklist, requires an explicit link between any clinical guideline and its primary evidence (available at www.agreetrust.org), the NG authors have not fully adhered to the AGREE process, although we note that our assessment is at odds with the AGREE evaluator who awarded 7/7 for this item (see on-line Supplement 3, available http://www.nature.com. ezproxy.library.uq.edu.au/articles/s41393-017-00173\#MOESM1).

Several unintended, adverse consequences may result from the acceptance and promotion of these NG, including: a. discouraging those who stand to gain most from commencing an exercise program, such as sedentary individuals with tetraplegia and/or multiple comorbidities. Many of these people will be unable to safely complete $20 \mathrm{~min}$ of continuous, moderate-intensity aerobic exercise and because the NG specifies that this is the minimum threshold for benefit, they will be given misleading impression that they will not benefit from doing smaller volumes;

b. creating the impression that people with SCI do not need to be as physically active as the general population in order to be healthy; and

c. causing a relative overestimation of the number of people with SCI who are sufficiently active for good health compared with the general population. This is a risk if the NG are used as the benchmark for differentiating active from inactive individuals with SCI because it stands to reason that the number of people with SCI meeting very-low volumes of exercise identified in the NG will be considerably larger than those meeting the ESSA recommendation (which is commensurate with recommendations for the general population). The fact that many people with SCI are so much less active than the general population is one of the most important reasons that promotion of physical activity among people with SCI should be an urgent priority. To make this case requires that the same standard is used to assess participation in people with and without SCI.

In conclusion, we urge readers to critically evaluate the primary evidence underpinning any clinical guideline/ recommendation. In relation to exercise for people with SCI, we believe the NG are based on a misinterpretation of the scientific evidence and that recommendations in the ESSA Position Statement are more defensible, being based on a balanced consideration of the best available scientific evidence.

\section{Compliance with ethical standards}

Conflict of interest SMT (Australia) was honoured to be invited to the International Meeting (23.11.16, Kelowna, Canada). SMT's opposition to the NG is faithfully recorded on the 8th page of the NG. On 8.4.17 SMT provided reasons for his opposition in writing to KMG and subsequently withdrew from the authorship team of the NG on the grounds of irreconcilable differences. The remaining authors declare that they have no conflict of interest.

\section{References}

1. Martin Ginis KA, van der Scheer JW, Latimer-Cheung AE, Barrow A, Bourne C, Carruthers P, et al. Evidence-based scientific exercise 
guidelines for adults with spinal cord injury: an update and a new guideline. Spinal cord. 2017.

2. Tweedy SM, Beckman EM, Geraghty TJ, Theisen D, Perret C, Harvey LA, et al. Exercise and sports science Australia (ESSA) position statement on exercise and spinal cord injury. J Sci Med Sport. 2017;20:108-15.

3. van der Scheer JW, Martin Ginis KA, Ditor DS, Goosey-Tolfrey VL, Hicks AL, West CR, et al. Effects of exercise on fitness and health of adults with spinal cord injury: A systematic review. Neurology. 2017:89:736-45.

4. Pelletier CA, Totosy de Zepetnek JO, MacDonald MJ, Hicks AL. A 16-week randomized controlled trial evaluating the physical activity guidelines for adults with spinal cord injury. Spinal cord. 2014:53:363-7.

5. Hicks AL, Martin KA, Ditor DS, Latimer AE, Craven C, Bugaresti $\mathrm{J}$, et al. Long-term exercise training in persons with spinal cord injury: effects on strength, arm ergometry performance and psychological well-being. Spinal Cord. 2003; 41:34-43.

6. Davis G, Plyley MJ, Shephard RJ. Gains of cardiorespiratory fitness with arm-crank training in spinally disabled men. Can J Sport Sci. 1991;16:64-72.

7. Kim DI, Lee H, Lee BS, Kim J, Jeon JY. Effects of a 6-week indoor hand-bike exercise program on health and fitness levels in people with spinal cord injury: a randomized controlled trial study. Arch Phys Med Rehabil. 2015;96:2033-40.e1.

8. Rosety-Rodriguez M, Camacho A, Rosety I, Fornieles G, Rosety MA, Diaz AJ, et al. Low-grade systemic inflammation and leptin levels were improved by arm cranking exercise in adults with chronic spinal cord injury. Arch Phys Med Rehabil. 2014;95:297-302.

9. Taylor AW, McDonell E, Brassard L. The effects of an arm ergometer training programme on wheelchair subjects. Paraplegia. 1986;24:105-14. 